Revue de droit comparé du travail et de la sécurité sociale

$2 \mid 2020$

La vie personnelle du salarié

\title{
Les droits de l'employeur sur la vie privée du salarié : étude du droit russe
}

Elena Serebryakova et Elena Sychenko

\section{(2) OpenEdition}

1 Journals

Édition électronique

URL : https://journals.openedition.org/rdctss/1095

DOI : 10.4000/rdctss. 1095

ISSN : 2262-9815

Éditeur

Centre de droit comparé du travail et de la sécurité sociale

Édition imprimée

Date de publication : 1 juin 2020

Pagination : 76-85

ISSN : 2117-4350

Référence électronique

Elena Serebryakova et Elena Sychenko, « Les droits de l'employeur sur la vie privée du salarié : étude du droit russe », Revue de droit comparé du travail et de la sécurité sociale [En ligne], 2 | 2020, mis en ligne le 01 novembre 2021, consulté le 11 novembre 2021. URL : http://journals.openedition.org/ rdctss/1095; DOI : https://doi.org/10.4000/rdctss.1095

\section{cc)}

Revue de droit comparé du travail et de la sécurité sociale est mise à disposition selon les termes de la Licence Creative Commons Attribution - Pas d'Utilisation Commerciale - Pas de Modification 4.0 International. 


\section{LES DROITS DE L'EMPLOYEUR SUR LA VIE PRIVÉE DU SALARIÉ : ÉTUDE DU DROIT RUSSE}

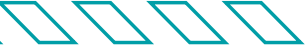

\section{Elena Serebryakova}

Maître de conférences, Université nationale de recherche «École supérieure d'économie », Moscou

\section{Elena Sychenko}

Maître de conférences, Université d'Etat de Saint-Petersbourg

\section{RÉSUMÉ}

Le Code du travail de la Fédération de Russie établit une distinction entre le temps de travail et le temps de repos. Cependant, le droit du salarié d'utiliser son temps de repos est souvent limité. Les restrictions sont établies par la loi et peuvent affecter le droit de l'employé de choisir une activité et sa forme. Dans les deux premières parties de cet article, nous examinons ces restrictions et leur application dans la jurisprudence russe. La troisième partie traite du contrôle du salarié pendant le travail.

Mots-clés : Fédération de Russie, temps de repos, emploi combiné, perte de confiance de l'employeur, acte immoral, la vidéosurveillance au travail

\section{ABSTRACT}

The Labour Code of the Russian Federation distinguishes between working time and rest time. However, the employee's right to use his rest time is often limited. The relevant restrictions are established by law and may affect the right of the employee to choose an activity and its form. In the first two parts of this article we examine these restrictions and their application in Russian case-law. The third part deals with the control over the employee during work.

Key words: Russian Federation, Rest Time, Combining Job, Loss of Confidence of the Employer, Immoral Deed, Video Surveillance at Work 
e Code du travail de la Fédération de Russie distingue les notions de temps de travail et de temps libre. Aux termes de l'article 106 du Code du travail, le temps libre correspond au «temps pendant lequel le salarié est libre de l'exercice de ses fonctions et qu'il peut utiliser à sa discrétion ». Toutefois, le droit du travailleur à utiliser son temps libre est souvent limité. Les restrictions en la matière sont établies par la loi et peuvent affecter le droit du salarié de choisir une activité et sa forme.

Ces restrictions portent sur l'exercice d'une autre activité professionnelle pendant le temps libre (I) ou sur l'exercice de tout autre activité personnelle en dehors du lieu et du temps de travail (II). Elles peuvent aussi être exercées sur le lieu de travail (III).

\section{I - LES RESTRICTIONS À L'EXERCICE D'ACTIVITÉS PROFESSIONNELLES SUR LE TEMPS LIBRE}

La législation du travail russe permet à un salarié de conclure différents contrats de travail avec d'autres employeurs pendant son temps libre, en dehors de son travail principal (Article 282 du Code du travail de la Fédération de Russie). La restriction de ce droit reste néanmoins possible pour les raisons suivantes:

1- Protection de la santé des salariés. Cette raison est à la base de l'interdiction du travail secondaire ${ }^{1}$ pour les personnes âgées de moins de 18 ans, et de l'interdiction de ce travail dans des conditions dangereuses ou nocives, si le travail principal s'exerce déjà dans ces mêmes conditions ;

2 - Protection des intérêts publics, qui justifie l'interdiction faite aux travailleurs employés dans les transports (conducteurs de bus, train, métro, etc.) d'exercer des activités similaires en tant que travail secondaire (article 329 du Code du travail) ;

3 - Protection des intérêts des entreprises. Dans ce cadre, le Code du travail définit le travail secondaire pour le sportif (article 348.7 du Code) et pour le chef d'entreprise (article 276 du Code). En effet, ceux-ci ne peuvent exercer un travail principal et un travail secondaire pour des entreprises concurrentes sans autorisation de l'employeur principal ;

4 - Protection des intérêts de l'Etat. À cette fin, des restrictions au travail secondaire sont imposées aux fonctionnaires d'Etat et territoriaux ainsi qu'aux procureurs ${ }^{2}$. Pour autant, les personnes appartenant à ces catégories ont le droit de s'engager, durant leur temps libre, dans des activités telles que la recherche scientifique et l'enseignement.

1 Le travail secondaire est un travail effectué durant le temps libre laissé par le travail principal du salarié.

2 Article 17 de la Loi fédérale du 27/07/2004 n79-FZ «Sur le service civique de la Fédération de Russie » (Federalniy zakon " O gosudarstvennoy grazhdanskoy sluzhbe Rossiyskoy Federatsii »); Article 14 de la Loi fédérale du 2/03/2007 n²5-FZ « Sur le service municipal dans la Fédération de Russie » (Federalniy zakon « O munitsypalnoy sluzhbe v Rossiskoy Federatsii »); Article 4 de la Loi fédérale du 17/01/1992 n²201-1 «Sur le parquet de la Fédération de Russie » (Federalniy zakon « O prokurature Rossiyskoy Federatsii »). 
Le Code du travail ne prévoit pas la possibilité de restreindre les droits à l'emploi d'un ancien collaborateur après la résiliation de son contrat de travail. Il ne reconnait pas non plus l'accord de non-concurrence. Selon le Ministère du travail de la Fédération de Russie, un tel accord limitant la liberté de travail d'un salarié viole l'article 37 de la Constitution de la Fédération de Russie, ainsi que la législation du travail³.

Selon l'article 55 de la Constitution de la Fédération de Russie, la restriction des droits et libertés des citoyens (en l'occurrence le droit au travail) n'est possible que dans le cadre de la protection des droits et intérêts des autres personnes, définie par la législation en vigueur. Jusqu'à présent, en dépit des affirmations de certains experts ${ }^{4}$ sur la nécessité d'autoriser les accords de non-concurrence, de telles règles n'ont pas été adoptées et la conclusion de ce type d'accords avec les professionnels reste illégale.

Dans la pratique, cette approche justifie les rejets de demandes d'indemnités compensatrices émanant d'ex-salariés signataires d'un accord de non-concurrence. En particulier, la Cour a considéré illégal un tel accord, car l'article 9 alinéa 2 du Code du travail prévoit que les contrats de travail ne peuvent contenir des conditions et des restrictions aux droits, ou la réduction du niveau de garanties pour les salariés, par rapport à celles établies par la législation du travail. La Cour a ainsi refusé le paiement des indemnités compensatrices au salarié 5 .

Toutefois, dans certains cas, les anciens fonctionnaires sont soumis à autorisation pour l'exercice d'un autre emploi. Cette règle est prévue par l'article 17 de la Loi « sur le service civique de la Fédération de Russie ». Dans les deux années qui suivent son départ ${ }^{6}$, un fonctionnaire n'a pas le droit de travailler, sans le consentement de la Commission spéciale, dans des organisations à caractère commercial ou à but non lucratif s'il était en relation avec elles durant ses attributions professionnelles antérieures. La violation de cette interdiction entraîne le licenciement de l'ex-fonctionnaire de son nouveau poste. Dans ce cadre, le Procureur peut être l'initiateur de la résiliation du contrat de travail?.

\section{II - LES RESTRICTION D'ACTIVITÉS NON PROFESSIONNELLES DURANT LE TEMPS LIBRE}

La législation russe prévoit le licenciement d'un salarié au regard de ses activités dans le cadre du temps libre de sa vie personnelle lorsque ces activités entrainent une perte de confiance de l'employeur (A) ou si le salarié a commis une faute immorale (B).

3 Lettre d'information du Ministère du travail et de la protection sociale de la Fédération de Russie du 19 octobre 2017 n¹4-2/B-942.

4 Voir S. Vasilyeva, "Travail sur deux fronts : quand un top manager se rend chez un concurrent" [Rabota na dva fronta: kogda top ukhodit $k$ konkurentu], Trudovoe pravo, 2018, n¹, p. 75 ; A. V. Zavgorodniy, « Accord de non-concurrence : expérience des pays étrangers » [Soglachenie o nekonkurentsii s rabotnikami (zarubezhniy opyt)], Peterburgskiy yurist, 2016, n³, p. 73.

5 Jugement en appel de la Cour de Moscou du 2 juin 2017, n³3-20918/2017.

6 La liste des postes est établie par le décret du Président de la Fédération de Russie du 21/07/2010 $n^{\circ} 925$ «Sur les mesures visant à mettre en œuvre certaines dispositions de la loi fédérale "Sur la lutte contre la corruption" ».

7 Voir par exemple la décision du tribunal de l'arrondissement Meschansky de Moscou du 4 mai $2017 n^{\circ} 2-7290 / 2017$. 


\section{A - LES ACTIVITÉS ENTRAînANT UNE PERTE DE CONFIANCE DE L'EMPLOYEUR}

La perte de confiance est un motif de rupture du contrat de travail du salarié, d'un fonctionnaire d'Etat ou d'un fonctionnaire territorial. Pour licencier un salarié en invoquant ce motif, l'employeur doit prouver qu'il a commis une infraction. Le salarié peut être licencié même si ladite infraction n'est pas liée à son travail et est commise en dehors de l'entreprise ${ }^{8}$. Ce motif de licenciement est applicable uniquement aux salariés qui sont responsables de valeurs monétaire ou marchandes, par exemple cassiers, agents transitaires, vendeurs...

La perte de confiance à l'égard d'un fonctionnaire peut être engendrée par le non-respect des interdictions et restrictions définies dans le droit de la fonction publique. Les restrictions et interdictions non compatibles avec les règles de la fonction publique sont nombreuses. Il s'agit notamment de l'interdiction de déclarations publiques, de jugements et d'évaluations des activités d'autorités de la fonction publique et de leurs dirigeants, y compris les décisions d'une autorité publique supérieure, ou d'une autorité publique dans laquelle le fonctionnaire assure un remplacement si cela ne relève pas directement de ses fonctions statutaires?.

Cette interdiction, appelée interdiction de critiquer les décisions de la hiérarchie et critiquée par de nombreux spécialistes ${ }^{10}$, est reconnue comme conforme à la Constitution de la Fédération de Russie. La conclusion en a été tirée par la Cour constitutionnelle en 2011, suite à des plaintes formulées par des fonctionnaires de la police et de l'administration fiscale licenciés pour avoir critiqué les modalités de calcul de leurs salaires.

Tout en reconnaissant cette interdiction comme étant conforme à la Constitution, la Cour constitutionnelle a indiqué qu'elle ne pouvait être considérée comme interdisant aux fonctionnaires d'exprimer leur opinion, jugement et appréciation sur les activités des autorités publiques. Pour apprécier la légalité de l'action d'un fonctionnaire, un certain nombre de circonstances doivent être prises en compte : le contenu des déclarations publiques, leur intérêt, le rapport entre les dommages causés à l'intérêt public et les dommages évités, la possibilité d'un fonctionnaire de défendre ses droits ou l'intérêt public par d'autres moyens que la déclaration.

Toutefois, l'analyse de la jurisprudence relative à l'application des dispositions législatives en cause montre que certaines Cours indiquent encore dans leurs décisions que « le simple fait qu'un policier ait fait publiquement une déclaration dans les médias pour critiquer les activités du chef des services de l'Intérieur est une raison suffisante pour lui infliger des sanctions disciplinaires ${ }^{11}$.

8 Alinéa 45 de la Résolution de l'Assemblée plénière de la Cour Suprême de la Fédération de Russie du 17/03/2004 n².

9 Article 17 alinéa 1 paragraphe 17 de la Loi fédérale « Sur le service civique de la Fédération de Russie ".

10 Voir M. V. Presnyakov et S. E. Tchanov, «Liberté d'expression dans la fonction publique : le démantèlement achevé " [Svoboda slova na gosudarstvennoy grazhdanskoy sluzhbe : demontazh zaverchen], Administrativnoe pravo y protsess, 2017, n¹1, p. 18.

11 Voir la décision du tribunal de la ville de Noyabrsk du 20 février 2017 n²-405/2017. 
Une telle approche élimine pratiquement toute obligation de transparence de la part des pouvoirs publics et se trouve manifestement en contradiction avec la Recommandation du Conseil de l'Europe relative à la protection des lanceurs d'alerte.

En effet, la Cour européenne des droits de l'homme a déclaré, à plusieurs reprises, que dans un système démocratique, les actions ou omissions du Gouvernement doivent se trouver placées sous le contrôle attentif non seulement des pouvoirs législatif et judiciaire, mais aussi des médias et de l'opinion publique ${ }^{12}$.

\section{B - LA FAUTE IMMORALE DU SALARIÉ}

Une faute immorale commise pendant son temps libre peut être à l'origine du licenciement d'un salarié qui exerce des fonctions éducatives, comme par exemple un enseignant, un professeur, un maître de formation professionnelle. Le Code du travail ne prévoit pas de définition du caractère immoral d'une faute. Dans chaque affaire, la question de l'immoralité des actions de l'enseignant est tranchée au cas par cas.

Le Ministère de l'éducation a élaboré des normes éthiques professionnelles pour les enseignants ${ }^{13}$, qui sont notamment encouragés à :

- éviter les situations susceptibles de porter atteinte à l'honneur, la dignité et la réputation d'un enseignant ou d'un établissement d'éducation ;

- s'abstenir de diffuser sur Internet et dans des lieux accessibles aux enfants, des informations préjudiciables à leur santé ou à leur développement.

La nature des informations préjudiciables à la santé ou au développement des enfants est définie par la loi ${ }^{14}$.

Il s'agit notamment des informations :

- susceptibles d'inciter les enfants à consommer des stupéfiants, du tabac, de l'alcool, à se prostituer, à vagabonder ou à mendier ;

- justifiant un comportement illégal ou la licéité de la violence, ou incitant à la violence à l'égard des êtres humains ou des animaux ;

- encourageant des relations sexuelles;

- contenant des mots grossiers ou vulgaires.

La diffusion de ces informations par l'enseignant est considérée, par son employeur, comme une action incompatible avec la poursuite d'activités éducatives; il en est de même, si l'enseignant, pendant son temps libre, a publié sur sa page privée d'un réseau social des photos sur lesquelles, par exemple, il fume ou consomme de l'alcool. L'analyse de la jurisprudence montre que dans ces situations, les tribunaux valident la décision de l'employeur et refusent la réintégration des enseignants concernés.

12 CEDH, affaire Guja c. Moldova ( $n^{\circ}$ 14277/04) du 12 février 2008, alinéa 74.

13 Lettre du Ministère de l'éducation et du syndicat des salariés de l'enseignement public et des sciences de la Fédération de Russie du 20/08/2019 « Modèle de règlement sur l'éthique professionnelle des enseignants ».

14 Article 5 de la Loi fédérale du 29/12/2010 n436-FZ « Sur la protection des enfants contre les informations préjudiciables à leur santé et à leur développement » [Federalniy zakon "O zaschite detey ot informatsii, pritchinyayuschey vred ikh zdorovyu i razvitiyu »]. 
Les prescriptions relatives à l'apparence de l'enseignant ne sont pas vraiment régies par les règles éthiques professionnelles de l'enseignement. Cependant, les enseignants sont encouragés à « adhérer à une apparence conforme aux objectifs du programme éducatif en cours ». De plus, les employeurs exigent que les salariés dans la sphère éducative suivent cette règle, tant au travail que dans leur vie privée. La publication de photos au contenu «frivole » sur les réseaux sociaux peut être la cause de licenciement, l'évaluation de la «frivolité » de l'image restant à la discrétion de l'employeur.

Au cours des dernières années, de nombreuses enseignantes ont ainsi failli perdre leur emploi en raison de la publication sur les réseaux sociaux de photos en maillot de bain, y compris lors de compétitions de natation. L'épisode particulièrement notable fut le cas d'une enseignante qui, durant son temps libre, a suivi une formation dans une agence de mannequinat, durant laquelle elle a participé à une séance photo en maillot de bain de style rétro. L'agence ayant publié ces photos sur Internet, la direction de l'école où travaillait l'enseignante a estimé que son comportement portait attente à l'image de l'établissement.

Dans ce cas et dans bien d'autres, les salariés n'ont pas cherché à se défendre devant les tribunaux, mais c'est le soutien des parents et de l'opinion publique qui a contribué à leur retour au travail dans les écoles.

\section{III - LE CONTRÔLE DE LA VIE PRIVÉE SUR LE LIEU DE TRAVAIL}

Contrairement à la législation de plusieurs pays européens ${ }^{15}$, la législation russe ne contient pas de règles spéciales relatives à la procédure de surveillance du travailleur ou relatives aux garanties de sa vie privée sur le lieu de travail. Néanmoins, le droit au respect de la vie privée, au secret de la correspondance, des conversations téléphoniques et des mails, sont garantis par l'article 23 de la Constitution de la Fédération de Russie.

Il est ici utile d'analyser la jurisprudence en matière de recours exercés par des salariés contre la vidéo-surveillance utilisée pour vérifier les processus de travail. II semble que les tribunaux aient tendance à ignorer les règles sur le droit au respect de la vie privée, dans la mesure où ils concluent par exemple que « la vidéosurveillance a été réalisée sur le lieu de travail, de sorte que les salariés de l'entreprise et le demandeur n'ont pas été lésés dans la vie privée ${ }^{16}$, ou encore que "l'utilisation de la vidéo-surveillance par l'employeur ne viole pas les droits constitutionnels du salarié au respect de la vie privée (...) si elle est effectuée à des fins liées à la protection du bâtiment et non pour établir les circonstances de sa vie privée $»^{17}$.

15 Par exemple, en Italie, l'article 4 de la loi n³00/1970 sur les travailleurs traite de la procédure d'utilisation des moyens techniques pour le contrôle du travailleur; en Espagne, l'article 18 de la loi sur les travailleurs traite notamment de la vie privée du travailleur (voir le décret législatif Royal $n^{\circ} 2 / 2015$ du 23 octobre 2015).

16 Décision du tribunal de district Volzhsky de la ville de Saratov du 20 juillet 2012 n²-3212.

17 L'affaire concerne la surveillance vidéo des salariés, et le licenciement sur la base des données des caméras vidéo installées dans le bâtiment : Décision du tribunal de District de Moscou de Riazan du 29 avril $2019 n^{\circ} 2-2607 / 2018$. Pour une approche similaire en ce qui concerne les caméras installées pour assurer la sécurité dans une entreprise, voir notamment : le jugement en appel de la Cour de la région de Krasnoïarsk du 14 novembre 2012 n³3-989920; la décision de tribunal de la ville de Michurinsk de la région de Tambov du 15 juillet 2016 n²-947/2016. 
Il est intéressant de noter que les vidéos enregistrées par les caméras installées pour la "sécurité " ont pourtant été utilisées par certains employeurs pour prouver l'absence d'un salarié sur le lieu de travail. Dans d'autres affaires, les tribunaux laissent sans réponse l'argument du salarié selon lequel la vidéo viole son droit au respect de la vie privée ${ }^{18}$.

En raison de l'absence de règles spécifiques concernant la procédure de contrôle des travailleurs, la réglementation des enregistrements vidéo et audio, ainsi que la collecte d'informations par des moyens techniques, doivent être effectuées conformément à la Loi «Sur les données personnelles». Ce sont les dispositions de cette loi qui donnent souvent lieu à un recours du salarié contre la surveillance vidéo établie par l'employeur, ou à une contestation de la sanction imposée pour une faute établie à la suite de la collecte d'informations par l'employeur par des moyens techniques.

S'agissant de la procédure de traitement des données personnelles, établie par la loi susmentionnée, une personne peut décider de fournir, dans son intérêt propre, ses données personnelles (article 9 de la loi). Le consentement pour leur utilisation doit être libre et spécifique, sans pression d'aucune sorte, et la personne concernée doit en être préalablement informée.

Une réglementation spéciale est prévue pour le traitement des données biométriques, c'est-à-dire des informations qui définissent les caractéristiques physiques et biologiques d'une personne, sur la base desquelles il est possible d'établir son identité. Étant donné que l'enregistrement vidéo, la photographie et l'enregistrement audio, permettent d'établir l'identité du travailleur, ces contrôles de collecte de données personnelles biométriques nécessitent un consentement écrit (article 11 de la loi). Dans ce consentement, il est important d'indiquer, en particulier, le but du traitement des données personnelles, leur énumération et la liste des actions pour lesquelles le consentement est donné, ainsi que la méthode de retrait du consentement (article 9 de la loi).

L'analyse de la jurisprudence démontre que, dans la plupart des cas, les tribunaux, ne sont pas enclins à considérer la vidéo comme une méthode permettant d'obtenir les données biométriques du salarié. Ils négligent souvent l'argument du salarié relatif à l'absence d'un consentement écrit autorisant le traitement des données ${ }^{19}$. Ces mêmes tribunaux considèrent comme consentement écrit la signature du salarié apposée sur un document indiquant qu'il a pris connaissance du règlement relatif à l'utilisation de ses données personnelles par l'entreprise ${ }^{20}$.

Selon une autre tendance évidente, les tribunaux russes estiment que «l'enregistrement vidéo n'est pas une divulgation des données personnelles d'un salarié et ne viole pas les exigences de la loi $»^{21}$. En règle générale, les juges ne donnent pas d'arguments juridiques en faveur de cette interprétation de la loi.

18 Voir le cas de l'installation sur un ordinateur professionnel du programme «Cliché instantané » qui garde la trace de tous les fichiers : décision du tribunal de district Leninsky de la ville de Yaroslavl du 27 mars $2019 n^{\circ} 2-1688 / 2018$.

19 Décision du tribunal de district Partizansky (Primorsky Krai) du 15 juin 2018 n²-466/2018; décision du tribunal de la ville de Chouya du 30 mai 2016 n²-1834/2016.

20 Décision du tribunal d'arrondissement Presnensky de Moscou du 2 juin 2015 n²-19/2015.

21 Décision du tribunal de district Moskovsky de la ville de Ryazan du 29 avril 2019 n²-2607/2018 ; jugement en appel de la Cour de la région d'Orenbourg du 3 décembre 2014 n³3-7039/2014. 
Dans la pratique, seules deux affaires ont conduit le tribunal à interpréter littéralement la loi et à appliquer strictement ses dispositions, concluant ainsi que l'enregistrement vidéo était illégal. Dans la première affaire, la décision était motivée par l'absence de consentement écrit du salarié22, alors que dans la seconde, l'utilisation des vidéos reçues a été déclarée non-conforme aux objectifs définis dans le consentement signé par le salarié23.

L'analyse de la pratique jurisprudentielle sur la surveillance vidéo du salarié montre que l'enregistrement vidéo, comme les autres contrôles portant sur les obligations professionnelles du salarié effectués à l'aide de moyens techniques -tels que la copie de clichés instantanés de tous les fichiers sur l'ordinateur du salarié24-, sont interprétés par les tribunaux comme la réalisation du droit de l'employeur à la gestion du travail (article 22 du Code du travail).

En règle générale, l'existence d'un consentement général pour le traitement des données personnelles ou la fixation dans le règlement interne de l'entreprise de la possibilité d'enregistrement vidéo du processus de travail est considérée comme suffisante pour la reconnaissance légale d'un tel contrôle ${ }^{25}$.

Enfin, une caractéristique importante de la jurisprudence russe est le fait que, dans la plupart des cas, les plaignants licenciés pour manquement à des obligations professionnelles, prouvé par enregistrement vidéo, ne contestent pas l'illégalité de cette preuve et la violation de leur droit au respect de la vie privée ${ }^{26}$. Dans aucune de ces affaires, les tribunaux n'ont enquêté sur la légalité de l'enregistrement vidéo, en dépit de l'article 55 du Code de procédure civile énonçant que les preuves, reçues en violation de la loi, n'ont pas de force juridique et ne peuvent servir de base à la décision du tribunal.

L'analyse des décisions des tribunaux russes sur l'ingérence de l'employeur dans la vie privée du salarié et sur le traitement des données personnelles, permet de mettre en lumière les approches systémiques suivantes:

- une approche large pour déterminer la portée du pouvoir discrétionnaire de l'employeur et déterminer les méthodes de contrôle des travailleurs sur le lieu de travail;

- l'examen des affaires par le juge à la lumière de la protection des données personnelles, sans prise en considération de la protection du droit au respect de la vie privée;

- l'interprétation du consentement général du salarié à l'utilisation de ses données personnelles donné dans le cadre du contrat de travail, comme le consentement à tout type de contrôle de l'employé via l'utilisation de diverses techniques de surveillance et technologies de l'information;

- la non-application des normes sur la nécessité du consentement écrit du salarié dans le traitement des données personnelles biométriques.

22 Décision de la Cour suprême de la République du Daghestan du 24 mai 2018.

23 Décision du tribunal de district Centralny de la ville de Sotchi du 21 février 2013 n²-780/2013.

24 Décision du tribunal de District de Leninski de la ville de Yaroslavl du 27 mars 2019 n²-1688/2018.

25 Décision du tribunal de district Privokzalny de la ville de Toula du 26 juin $2017 n^{\circ} 2-502 / 2017$; Décision du tribunal de district de Leninsky de la ville de Voronej du 19 mai 2017 n²-1609/2017; Jugement en appel de la Cour de la région de l'Altaï du 15 octobre 2013 n³3-8403/2013.

26 Décision du tribunal de district Frunzensky de la ville de Yaroslavl du 4 juillet 2019 n²-999/2019; décision du tribunal de district Uchalinsky (République de Bachkirie) du 21 juin 2019 n²-873/2019; décision du tribunal de district de Leninsky de la ville de Novossibirsk du 19 juin 2019 n²-1571/2019. 
Force est de constater que dans la plupart des cas d'utilisation de la vidéo lors du processus de travail, les tribunaux appliquent incorrectement - ou n'appliquent pas - la loi fédérale "Sur les données personnelles».

En outre, les tribunaux se montrent sceptiques quant à l'existence d'une « vie privée » sur le lieu de travail, ignorant de fait les arguments des salariés portant sur la violation par l'employeur de ce droit constitutionnel. Cette position est une manifestation de l'hérédité soviétique. La période de I'URSS se caractérisait notamment par le flou des frontières entre la vie privée et publique, en dehors du temps de travail et par le déni sans équivoque de la vie privée sur le lieu de travail. L'évolution de la situation économique et politique n'entraîne pas automatiquement le renforcement de nouvelles valeurs dans la société, malgré leur incorporation dans la législation nationale.

Les exemples positifs d'autres pays et l'influence du droit international peuvent être importants dans ce processus. Selon l'alinéa 4 de l'article 15 de la Constitution de la Fédération de Russie, le droit international fait partie du système juridique russe et a priorité sur les normes nationales.

À cet égard, la position de la Cour européenne des droits de l'homme concernant les limites de la protection de la vie privée du travailleur, les facteurs à prendre en compte par les tribunaux dans les affaires de vidéo surveillance du travailleur ou d'ouverture de son courrier, revêt une importance particulière ${ }^{27}$. En outre, la connaissance par les avocats des positions juridiques de la CEDH sur la question de la protection de la vie privée sur le lieu de travail, et la référence dans de telles déclarations à l'article 8 de la Convention Européenne des Droit de l'Homme et aux décisions susmentionnées de la CEDH, pourraient entraîner un changement significatif de la pratique judiciaire en Russie.

Il convient de souligner qu'en général, la législation de la Fédération de Russie est conforme aux normes de la Convention ${ }^{28}$. Dans le même temps, la pratique établie de son utilisation est contraire non seulement aux exigences générales pour le traitement des données personnelles des salariés, établies par l'article 86 du Code du travail, mais également aux principes de base de la protection de la vie privée découlant de l'article 8 de ladite Convention.

\section{Conclusion}

Les droits de certaines catégories de travailleurs visant à choisir l'utilisation de leur temps libre sont restreints. Les restrictions apportées à l'exercice d'un travail à titre secondaire reposent sur la nécessité de protéger les intérêts du travailleur lui-même, de la société ou de l'employeur.

27 CEDH, affaire Bărbulescu c. Romanie (61496/08) 5 septembre 2017, alinéa 121; affaire Köpke c. Allemagne (420/07) du 5 octobre 2010; affaire Copland c. Royaume-Uni (62617/00) du 3 avril 2007; affaire Peev c. Bulgarie (64209/01) du 26 juillet 2007.

28 Voir notamment la Loi fédérale du 27/07/2006 n 152-FZ "Sur les données personnelles » (Federalniy zakon «O personalnykh dannykh»). 
Les restrictions morales imposées au comportement des enseignants en dehors du lieu de travail offrent à l'employeur un large degré d'interprétation des activités de l'enseignant durant son temps libre.

Quant aux problèmes qui surviennent lors de l'examen, par les tribunaux, d'affaires relatives aux contrôles du travailleur sur le lieu et durant le temps de travail, ils pourraient être corrigés par le recours, dans la jurisprudence russe, aux décisions de la Cour européenne des droits de l'homme. 